\title{
Hygiene along the continuum of care in the early post-natal period: an observational study in Nigeria
}

Yolisa Nalule ${ }^{1}$, Helen Buxton ${ }^{1}$, Erin Flynn², Olutunde Oluyinka ${ }^{3}$, Stephen Sara ${ }^{4}$, Oliver Cumming ${ }^{1}$ and Robert Dreibelbis ${ }^{1 *}$

\begin{abstract}
Background: Newborns delivered in healthcare facilities in low- and middle-income countries are at an increased risk of healthcare associated infections. Facility-based studies have focused primarily on healthcare worker behaviour during labour \& delivery with limited attention to hygiene practices in post-natal care areas and largely ignore the wide variety of actors involved in maternal and neonatal care.

Methods: This exploratory mixed-methods study took place in six healthcare facilities in Nigeria where 31 structured observations were completed during post-natal care, discharge, and the first 6 hours after return to the home. Frequency of hand hygiene opportunities and hand hygiene actions were assessed for types of patient care (maternal and newborn care) and the role individuals played in caregiving (healthcare workers, cleaners, nonmaternal caregivers). Qualitative interviews with mothers were completed approximately 1 week after facility discharge.

Results: Maternal and newborn care were performed by a range of actors including healthcare workers, mothers, cleaners and non-maternal caregivers. Of 291 hand hygiene opportunities observed at health facilities, and 459 observed in home environments, adequate hand hygiene actions were observed during only $1 \%$ of all hand hygiene opportunities. Adequate hand hygiene prior to cord contact was observed in only $6 \%(1 / 17)$ of cord contact related hand hygiene opportunities at healthcare facilities and 7\% (2/29) in households. Discharge advice was infrequent and not standardised and could not be remembered by the mother after a week. Mothers reported discomfort around telling non-maternal caregivers to practice adequate hand hygiene for their newborn.

Conclusions: In this setting, hand hygiene practices during post-natal care and the first 6 hours in the home environment were consistently inadequate. Effective strategies are needed to promote safe hand hygiene practices within the post-natal care ward and home in low resource, high-burden settings. Such strategies need to target not just mothers and healthcare workers but also other caregivers.
\end{abstract}

Keywords: Hand hygiene, Healthcare associated infections, Post-natal, Neonatal infection, Infection prevention and control, Newborn care, Nigeria, Maternal infection

\footnotetext{
* Correspondence: Robert.Dreibelbis@lshtm.ac.uk

'Disease Control Department, London School of Hygiene and Tropical Medicine, London WC1E 7HT, UK

Full list of author information is available at the end of the article
}

(c) The Author(s). 2020 Open Access This article is licensed under a Creative Commons Attribution 4.0 International License, which permits use, sharing, adaptation, distribution and reproduction in any medium or format, as long as you give appropriate credit to the original author(s) and the source, provide a link to the Creative Commons licence, and indicate if changes were made. The images or other third party material in this article are included in the article's Creative Commons licence, unless indicated otherwise in a credit line to the material. If material is not included in the article's Creative Commons licence and your intended use is not permitted by statutory regulation or exceeds the permitted use, you will need to obtain permission directly from the copyright holder. To view a copy of this licence, visit http://creativecommons.org/licenses/by/4.0/. The Creative Commons Public Domain Dedication waiver (http://creativecommons.org/publicdomain/zero/1.0/) applies to the data made available in this article, unless otherwise stated in a credit line to the data. 


\section{Background}

In populations with a high burden of neonatal mortality, up to half of all neonatal deaths are caused by infections, many of which are transmitted at the time of childbirth [1,2]. Facility-based births are essential to providing safe, quality healthcare to mothers and newborns at the time of childbirth. However, newborns born in healthcare facilities (HCF) in low- and middle-income countries (LMIC) are at an increased risk of early onset sepsis due to unhygienic care practices during childbirth and post-natal care [3, 4]. Estimates suggest that newborns delivered in HCF in LMIC have 3-20 times greater risk of healthcare associated infections (HCAI) compared to newborns delivered in facilities in high income countries $[3,5]$. However, this gap is likely to widen, with increasing proportions of women in LMIC giving birth at HCFs lacking robust infection prevention and control and hygiene management practices $[1,5-8]$.

Hand hygiene practices are an essential component of infection prevention and control (IPC) strategies in newborn and maternal care [9-11]. Improved handwashing practices by birth attendants and mothers have been associated with a $19 \%$ and $44 \%$ reduction in neonatal mortality, respectively [9]. To promote adequate hand hygiene in healthcare settings globally, the World Health Organisation (WHO) has published hand hygiene guidelines for healthcare workers $(\mathrm{HCW})$ on key moments for hand hygiene during patient care [12-14]. Hand hygiene is also included as part of WHO recommended essential practices during newborn care - specifically before and after handling the newborn, before and after cord care, and after diaper changing [15].

Many HCF-based studies on hand hygiene practices during maternal and newborn care in LMIC have focused on HCW hand hygiene during labour and delivery or high-risk environments such as the neonatal intensive care units [16-20]. Community-based studies have generally focused on caregiver hygiene practices for newborns who are born outside the health facility or during the late post-natal period ( $>7$ days after birth) [18, 21-25]. Limited attention has been given to understanding hand hygiene compliance by the wide range of personnel and caregivers providing facility- and home-based newborn care in the immediate and early post-natal period ( $<8$ days after birth) $[26,27]$. Understanding hand hygiene practices of various caregivers during this early post-natal period and how these are influenced by multiple factors allows for the design and implementation of more effective facility-based interventions to improve behaviours. The objective of this study is to document observed hand hygiene practices during the early post-natal care period in the healthcare facility and the first 6 hours after returning home.

\section{Methods}

\section{Study design}

The data presented here were collected over 4 weeks in July 2017 as part of a larger mixed-methods study investigating hygiene practices during childbirth, post-natal care, and return to the home environment across six health facilities in Kogi and Ebonyi states. Data reported here focus on the post-natal care ward, facility discharge, and the home environment. Findings related to IPC standards and infrastructure and practices during labour and delivery have been reported elsewhere [28, 29].

Facilities sampled for this study were all participating in the Maternal and Child Survival Program (MCSP) quality of care improvement program funded by the United States Agency for International Development (USAID). While all participating facilities received interventions aimed at improving the quality and utilisation of maternal and neonatal healthcare services, focus on hygiene was very limited. Further details of the MCSP quality of care improvement programme and facility selection are described in previous publications [28, 29]. In both Kogi and Ebonyi states, we sampled one facility at each of three levels: one primary HCF, a secondary $\mathrm{HCF}$, and a tertiary HCF.

\section{Data collection}

Facility-level data collection has been previously described [28, 29]. In brief, a structured facility observational checklist and a needs assessment survey were completed on the first day of observation in each participating HCF.

\section{Structured observations}

Structured observations were completed during childbirth, post-natal care, discharge, and the first 6 hours after returning home. A total of 39 women were recruited across the six facilities for at least one observation period. As a descriptive exploratory study, sample size was based primarily on resources availability. Participant eligibility criteria and observations of hand hygiene during childbirth have been previously reported [28]. Post-natal care observations began when the mother and baby were moved from the delivery area to the maternity/post-natal ward, hereafter referred to as post-natal care ward. Data collection staff documented observed hygiene practices and actions of all individuals involved in any maternal and newborn care for a period of up to four continuous hours or until the mother was discharged.

At the time of discharge, data collection staff directly observed and documented the discharge process with emphasis on recording discharge instructions the new mother received, particularly any reference to hygiene, handwashing and cord care. After discharge, staff 
accompanied the woman and newborn to her home to observe the hand hygiene practices there. Home observations lasted from the time of arrival in the home for up to 6 hours. At the home, key observations included newborn care practices (breastfeeding, bathing, diaper changes and handling), hand hygiene practices of all individuals involved in any newborn care, and other standard hand hygiene opportunities (feeding of self or others, visiting toilet, food preparation). At the end of the home observation, a structured spot check was completed of the home environment to assess the presence and availability of water, sanitation, and hygiene facilities in the home.

\section{Semi structured interviews}

The questionnaire used, data collection methods and analysis for these interviews have been previously published [29]. Semi-structured interviews were completed with one facility head, nurse or midwife, cleaner and mother per participating facility for a total of $18 \mathrm{HCF}$ staff and 6 mothers. Mothers who consented to a second home visit had their phone numbers recorded at the end of the home observation. On the fifth day following the home visit, a selected mother was called to arrange the 45-min interview, which was then conducted in person at her home, at least 7 days after the initial visit. All household interviews were audio recorded and conducted in Yoruba, Igbo or English in two teams of two female local enumerators (an interviewer and a note taker), with prior experience of conducting qualitative research.

\section{Data analysis}

All quantitative data were analysed using StataSE 15 (Stata Corp, College Station, TX, USA). Data from the facility needs assessment and walk through tools were examined descriptively to provide context for structured observation findings. Qualitative notes recorded during the observation data collection were reviewed and where applicable, recoded using STATA.

Observation data analysis was descriptive and focused on frequency and sequence of hand hygiene opportunities and associated hand hygiene actions based on WHO's Five Moments for Hand hygiene and the three moments adapted for community neonatal hand hygiene $[30,31]$. For the purposes of analysis, hand hygiene "opportunities" were defined as any activity that put hands at potential risk of contamination or activities that resulted in possible transmission of infectious agents to the mother and/or newborn during the observed period. Hand hygiene opportunities related to maternal care included: conducting clinical procedures on mothers (intramuscular injections, intravenous (IV) procedures), changing of perineal pads and emptying urine pots.
Hand hygiene opportunities related to newborn care included direct cord contact via cord cleaning or cord inspection as well as activities during newborn care that could result in unobserved cord contact such as changing nappies, changing the newborn's clothes, and skin contact with the newborn's body. Hand hygiene "actions" were defined as any action taken in response, proactively or reactively, to a hygiene opportunity in an effort to mitigate potential infection transmission. Observed hand hygiene actions associated with each hand hygiene opportunity were coded into three categories in analysis. First, no action or action was assigned to any hand hygiene opportunity when there was no observed hand hygiene action taken or action taken. Hand hygiene actions were further coded as adequate (handwashing with soap and water) or inadequate (wearing gloves without handwashing with soap or rinsing with water only).

A variety of individuals were observed taking part in maternal and newborn care; we refer to these individuals as actors in our analysis. At the HCF, actors were categorised into five groups: mothers, fathers, $\mathrm{HCW}$ (doctors, nurses and midwives), cleaners (employed by the HCF), and visitors - all individuals not employed by the HCF and not the child's mother or father. At the home, actors were categorised into three groups in analysis: mothers, fathers and nonparental caregivers. Non-parental caregivers included all other individuals who were observed engaging in the newborn caregiving activities at the home and included household members, relatives, and other non-family visitors. Our analysis explored the frequency of hand hygiene opportunities and hand hygiene actions by type of actor (mothers, fathers, $\mathrm{HCW}$, visitors, cleaners and non-parental caregivers), by patient care setting (HCF and home) and type of care provided (maternal and newborn care).

Qualitative data was transcribed into Microsoft Word (Redmond, Washington) and analysed in Microsoft Word and Excel (Redmond, Washington). Findings from HCF staff interviews around IPC related practices have been previously reported [29]; qualitative data reported here focus on responses around discharge information and newborn care in the home environment.

Any self-reported practices by the mothers were compared against structured observations results. Interview and field note transcripts were coded by one author and independently reviewed by another [29]. Thematic analysis was deductive, based on the hand hygiene moments for community newborn care [31] specifically; during newborn handling - before carrying or after bottom cleaning following defecation, and cord care/contact. 


\section{Results}

\section{Participant information}

A total of 39 mothers were recruited across the six facilities for at least one observation period; 31 mothers during labour and delivery, 31 mothers during post-natal period at the HCF, and 30 mothers at home. Eight mothers dropped out of the study after the post-natal care observations due to observation fatigue or nonconsenting household members. An additional 7 participants were recruited for facility discharge and home observations.

Mothers had similar characteristics across the observations and interviews. All participating mothers reported they were married with a mean age of 30 (range: 19-39), had 2 previous births (range: 0-6) and spent an average of $35 \mathrm{~min}$ travelling to the health care facility (range: 5-120). Fathers were present in 26/31 post-natal care observations and in 28/30 home observations.

\section{Postnatal care}

\section{Water, sanitation, and hygiene facilities}

Functioning handwashing facilities with soap were available in 2 of the 6 post-natal care wards; however, no material for hand drying was present. There was no other provision for handwashing within any of the post-natal care wards e.g. alcohol-based hand rub.

\section{Hand hygiene opportunities and action}

A total of 291 hand hygiene opportunities were observed during the post-natal period, $27 \%$ related to maternal care $(79 / 291)$ and $73 \%$ related to newborn care (212/ 291) (Table 1).

Visitors accounted for 37\% (103/291) of all observed hand hygiene opportunities. The majority (95\%) of visitors' hand hygiene opportunities were during newborn care activities. The remaining observed hand hygiene opportunities were among HCW (29\%), mothers (21\%), fathers $(13 \%)$ and cleaners $(2 \%)$.

Table 1 Observed hand hygiene opportunities and actions within post-natal care ward

\begin{tabular}{|c|c|c|c|c|}
\hline & \multirow{2}{*}{$\begin{array}{l}\text { Hand } \\
\text { Hygiene } \\
\text { Opportunities } \\
\text { n }\end{array}$} & \multicolumn{3}{|c|}{$\begin{array}{l}\text { Hand hygiene actions } \\
\text { n (\%) }\end{array}$} \\
\hline & & Adequate $^{a}$ & Inadequate $^{b}$ & No Action \\
\hline \multicolumn{5}{|l|}{ All observations } \\
\hline Mothers & 61 & $2(3)$ & $11(18)$ & $48(79)$ \\
\hline Fathers & 37 & $0(0)$ & $0(0)$ & $37(0)$ \\
\hline Healthcare workers & 84 & $1(1)$ & $12(14)$ & $71(84)$ \\
\hline Cleaners & 6 & $0(0)$ & $0(0)$ & $6(100)$ \\
\hline Visitors & 103 & $0(0)$ & $0(0)$ & $103(100)$ \\
\hline Total & 291 & $3(1)$ & $23(8)$ & $265(91)$ \\
\hline \multicolumn{5}{|l|}{ Maternal care $^{c}$} \\
\hline Mothers & 16 & $0(0)$ & $3(19)$ & $13(81)$ \\
\hline Fathers & 0 & $0(0)$ & $0(0)$ & $0(0)$ \\
\hline Healthcare workers & 57 & $1(2)$ & $6(10)$ & $50(88)$ \\
\hline Cleaners & 1 & $0(0)$ & $0(0)$ & $1(100)$ \\
\hline Visitors & 5 & $0(0)$ & $0(0)$ & $5(100)$ \\
\hline Total & 79 & $1(1)$ & $9(12)$ & $69(87)$ \\
\hline \multicolumn{5}{|l|}{ Newborn care ${ }^{d}$} \\
\hline Mothers & 45 & $2(4)$ & $8(18)$ & $35(78)$ \\
\hline Fathers & 37 & $0(0)$ & $0(0)$ & $37(100)$ \\
\hline Healthcare workers & 27 & $0(0)$ & $6(22)$ & $21(78)$ \\
\hline Cleaners & 5 & $0(0)$ & $0(0)$ & $5(100)$ \\
\hline Visitors & 98 & $0(0)$ & $0(0)$ & $98(100)$ \\
\hline Total & 212 & $2(1)$ & $14(7)$ & $196(92)$ \\
\hline
\end{tabular}

${ }^{a}$ Adequate hand hygiene action includes washing hands with soap and washing hands with soap and wearing clean gloves for aseptic procedures ${ }^{\mathrm{b}}$ Inadequate hand hygiene action includes rinsing hands without using soap or wearing gloves for aseptic procedures without handwashing with soap prior to donning gloves

${ }^{c}$ Maternal care includes contact by the healthcare workers, intramuscular injections, IV-related procedures, changing perineal pads, and emptying urine pan ${ }^{d}$ Newborn care includes direct cord contact via cord cleaning or cord inspection and newborn handling (changing newborn's diapers, cleaning newborns bottom following defecation, picking up and putting newborn down, rubbing newborn's body with body oils and powders, cleaning newborn's eyes, changing newborn's clothes, drying newborn with cloth, wiping newborn's face) 
Across all actors, no hand hygiene action was observed in relation to $91 \%(265 / 291)$ of hand hygiene opportunities. Half (13/26) of all observed hand hygiene actions were by HCW and the other half $(13 / 26)$ by mothers. No hand hygiene actions were conducted by fathers, visitors, or cleaners. Only 3 of 26 hand hygiene actions observed were categorized as adequate (handwashing with soap and water) - once by a HCW prior to inspecting a mother's perineal stitches and twice by mothers; prior to cord cleaning and prior to carrying the newborn. The remaining 23 hand hygiene actions were inadequate, and included HCWs wearing gloves without washing hands with soap prior to glove use (12/26) and mothers rinsing hands with water only $(11 / 26)$. Among $\mathrm{HCW}$, half $(6 / 12)$ of inadequate hand hygiene actions were during maternal care - mostly prior to IV related procedures including cannula insertion, changing IV therapy bags and inspecting the IV cannula site. Of the 212 hand hygiene opportunities observed during newborn care, $8 \%$
(17/212) were related to cord contact and the rest (195/ 212), were during other contact with the newborn (Table 2).

Cord contact in the post-natal care ward was made by multiple actors - mothers, HCWs, cleaners and visitors. The majority (13/17) of cord contact hand hygiene opportunities happened prior to cord cleaning and the rest (4/17) were during umbilical cord stump inspections. Across the 17 cord contact hand hygiene opportunities observed, hand hygiene actions were conducted 5 times, all of which were prior to cleaning of the newborn's cord. Only $1 / 5$ of hand hygiene actions was adequately performed.

\section{Discharge}

The average length of stay after birth across all six facilities was $35 \mathrm{~h}$ (range: 7-96 h). Standardized discharge procedures were reported in all but one HCF. Key informants reported that discharge procedures included

Table 2 Observed hand hygiene opportunities and hand hygiene actions related to newborn care in post-natal care wards

\begin{tabular}{|c|c|c|c|c|}
\hline & \multirow{2}{*}{$\begin{array}{l}\text { Hand } \\
\text { hygiene } \\
\text { opportunities } \\
\text { n }\end{array}$} & \multicolumn{3}{|c|}{$\begin{array}{l}\text { Hand hygiene actions } \\
\text { n (\%) }\end{array}$} \\
\hline & & Adequate $^{a}$ & Inadequate ${ }^{b}$ & No Action \\
\hline \multicolumn{5}{|l|}{ All observations } \\
\hline Mothers & 45 & $2(4)$ & $8(18)$ & $35(78)$ \\
\hline Fathers & 37 & $0(0)$ & $0(0)$ & $37(100)$ \\
\hline Healthcare workers & 27 & $0(0)$ & $6(22)$ & $21(78)$ \\
\hline Cleaners & 5 & $0(0)$ & $0(0)$ & $5(100)$ \\
\hline Visitors & 98 & $0(0)$ & $0(0)$ & $98(100)$ \\
\hline Total & 212 & $2(1)$ & $14(7)$ & $196(92)$ \\
\hline \multicolumn{5}{|l|}{ Cord contact ${ }^{\mathrm{c}}$} \\
\hline Mothers & 7 & $1(14)$ & $0(0)$ & $6(86)$ \\
\hline Fathers & 0 & $0(0)$ & $0(0)$ & $0(0)$ \\
\hline Healthcare workers & 7 & $0(0)$ & $4(57)$ & $3(43)$ \\
\hline Cleaners & 1 & $0(0)$ & $0(0)$ & $1(100)$ \\
\hline Visitors & 2 & $0(0)$ & $0(0)$ & $2(100)$ \\
\hline Total & 17 & $1(6)$ & $4(24)$ & $12(70)$ \\
\hline \multicolumn{5}{|l|}{ Other newborn care ${ }^{d}$} \\
\hline Mothers & 38 & $1(3)$ & $8(21)$ & $29(76)$ \\
\hline Fathers & 37 & $0(0)$ & $0(0)$ & $37(100)$ \\
\hline Healthcare workers & 20 & $0(0)$ & $2(10)$ & $18(90)$ \\
\hline Cleaners & 4 & $0(0)$ & $0(0)$ & $4(100)$ \\
\hline Visitors & 96 & $0(0)$ & $0(0)$ & 96 (100) \\
\hline Total & 195 & $1(1)$ & $10(5)$ & $184(94)$ \\
\hline
\end{tabular}

${ }^{a}$ Adequate hand hygiene action includes washing hands with soap and washing hands with soap and wearing clean gloves for aseptic procedures ${ }^{\mathrm{b}}$ Inadequate hand hygiene action includes rinsing hands without using soap or wearing gloves for aseptic procedures without handwashing with soap prior to donning gloves

${ }^{\mathrm{c}}$ Cord contact includes direct cord contact via cord cleaning or cord inspection

dOther newborn care includes newborn handling outside cord care (changing newborn's diapers, cleaning newborns bottom following defecation, picking up and putting newborn down, rubbing newborn's body with body oils and powders, cleaning newborn's eyes, changing newborn's clothes, drying newborn with cloth, wiping newborn's face) 
specific health information that should be provided to mothers at the time of discharge. However, 9/33 mothers did not receive any discharge advice at all from the midwives (Table 3).

When given, discharge advice covered both maternal and newborn care. Information on hand hygiene, typically related to newborn care; specifically cord care, baby handling and breastfeeding. During observations, approximately half (17/33) of mothers received advice on washing their hands before handling the baby and 70\% (23/33) received information on clean cord care, including washing hands before and after applying chlorohexidine, applying chlorohexidine exclusively on the cord, and placing the cord outside the diapers. However, in follow-up qualitative interviews, the only hand hygiene related discharge information that mothers could recall was involving breast feeding practices.

... First and foremost, I was told that it is not good for a breastfeeding mother to keep long nails, that it harbours dirt and that it is dangerous to the baby. Secondly, I must wash my hands before I breastfeed the baby. In fact, I must make sure that the environment where the baby stays is very clean. - Mother, tertiary facility

\section{Home observations}

The average duration of home observations was $5.1 \mathrm{~h}$ (range: 2.4-6.8 h) with an average of 101 hand hygiene opportunities recorded per observation (range: 30-180). Home observations started an average of $4 \mathrm{~h}$ after discharge from the facility (range: 0-21). Three out of thirty women were observed for more than 6 hours post discharge.

\section{Water sanitation and hygiene facilities}

Half $(16 / 30)$ of households had access to a water source within the home, 7 had access to an on plot water source, and 7 used a public shared water source. The majority (27/30) of households had stored water within their household at the time of the observation, and all but one (29/30) had soap at the household. Of the 30 households visited, 21 had a private latrine, 8 had access to a latrine shared with other households, and 1 did not have access to a latrine. Two-thirds of households (20/ 30) had a handwashing facility within the compound, but only 9 households with a handwashing facility had soap or another cleansing agent present at the site. There was an average of 15 non-parental caregivers observed across household observations (range: 3-39). Non-parental caregivers included household members, visiting relatives, and other visitors.

\section{Hand hygiene: opportunities and action}

All interviewed mothers knew both when and how hands should be washed in the home environment:

Before carrying my baby, I wash my hand, before carrying her and anytime I go to the toilet, I wash my hand before carrying my baby, even if I go to the kitchen to cook, I wash my hand even if I just go to urinate I wash my hand before carrying my baby. Mother, secondary facility

Table 3 Observed discharge advice given to mothers

\begin{tabular}{|c|c|c|c|c|}
\hline Topic & Advice & Primary $(N=10)$ & Secondary $(N=10)$ & Tertiary $(N=13)$ \\
\hline \multirow[t]{7}{*}{ Mother care } & Do not insert anything into the vagina & 2 & 5 & 8 \\
\hline & Take rest and sleep & 5 & 8 & 8 \\
\hline & Wash perineum daily and after faecal excretion & 5 & 6 & 8 \\
\hline & Change sanitary pads every 4 to $6 \mathrm{~h}$ & 5 & 7 & 8 \\
\hline & Wash used pads or dispose of them safely & 4 & 4 & 8 \\
\hline & Wash the body daily. & 5 & 9 & 8 \\
\hline & Avoid sexual intercourse until the perineum heals & 3 & 5 & 5 \\
\hline \multirow[t]{4}{*}{ Newborn care } & Wash hands before handling baby & 3 & 6 & 8 \\
\hline & How to care for the cord & 6 & 9 & 8 \\
\hline & Sleep under an insecticide treated net & 6 & 6 & 8 \\
\hline & Exclusive breastfeeding & 6 & 7 & 8 \\
\hline \multirow[t]{2}{*}{ Other } & Other advice & 4 & 7 & 4 \\
\hline & No advice & 4 & 0 & 5 \\
\hline \multirow[t]{4}{*}{ Drugs } & Pain relief & 9 & 9 & 12 \\
\hline & Antiseptic & 2 & 7 & 7 \\
\hline & Vitamin C & 8 & 8 & 9 \\
\hline & Vitamin B complex & 8 & 9 & 5 \\
\hline
\end{tabular}


When I want to take care of the cord, I will wash my hand because I will be bathing the baby. I will wash my hand before I carry the baby for bathing. Mother, primary facility

However, this knowledge was not reflected in practice. In only $1 \%(5 / 459)$ of all hand hygiene opportunities observed in the home environment was hand hygiene performed adequately and hands rinsed at another $3 \%$ $(12 / 459)$ of hand hygiene opportunities (Table 4). Mothers conducted the majority (15/17) of the hand hygiene actions.

Cord contact accounted for 6\% (29/459) of all hand hygiene opportunities; the majority (22/29) related to cord cleaning. Adequate hand hygiene was observed during 2 of 29 cord contact-related hand hygiene opportunities.

Non-maternal caregivers performed a variety of activities in the household, many of which put them at potential risk of transmitting infections to newborns during caregiving [see Additional file 1]. Over half of newborns (19/30) were bathed within the first 6 hours of their arrival to the home and bathing often involved multiple non-maternal caregivers. In one household, a newborn was bathed by 6 different non-maternal caregivers during the observation period. Following bathing,
8/19 newborns were rubbed with oils, in some cases mixed with different substances including; cassava flour mixed in red oil; black soap; palm kernel oil; shea butter; garlic and raw egg.

Despite the clear role of non-maternal caregivers in newborn care, mothers reported that asking any caregivers beyond fathers to wash hands was not feasible. Mothers noted that the caregivers would 'not be happy' or that they would 'become angry' if they were asked to wash their hands:

Some visitors are in a haste, when they come they do not wash their hand, they carry their baby, after they go - [if asked to wash hands] they will become angry. - Mother secondary facility

\section{I can't tell visitors like that! - Mother, tertiary facility}

Some mothers, however described strategies for protecting their newborn, mostly by using the baby wrap as a physical barrier between the skin and the non-parental caregivers' contaminated hands.

If I ask them to wash their hands, I don't know what they will feel! That is why I cover my baby with a

Table 4 Observed hand hygiene opportunities and hand hygiene actions in the household

\begin{tabular}{|c|c|c|c|c|}
\hline & \multirow{2}{*}{$\begin{array}{l}\text { Hand } \\
\text { hygiene } \\
\text { opportunities } \\
\mathrm{n}\end{array}$} & \multicolumn{3}{|c|}{$\begin{array}{l}\text { Hand hygiene actions } \\
\text { n (\%) }\end{array}$} \\
\hline & & Adequate $^{a}$ & Inadequate $^{b}$ & No Action \\
\hline \multicolumn{5}{|l|}{ All observations } \\
\hline Mothers & 154 & $4(3)$ & $11(7)$ & $139(90)$ \\
\hline Fathers & 7 & $0(0)$ & $0(0)$ & $7(100)$ \\
\hline Non-parental caregivers & 298 & $1(0.5)$ & $1(0.5)$ & $296(99)$ \\
\hline Total & 459 & $5(1)$ & $12(3)$ & $442(96)$ \\
\hline \multicolumn{5}{|l|}{ Cord contact $^{c}$} \\
\hline Mothers & 16 & $2(13)$ & $1(6)$ & $13(81)$ \\
\hline Fathers & 0 & $0(0)$ & $0(0)$ & $0(0)$ \\
\hline Non-parental caregivers & 13 & $0(0)$ & $0(0)$ & $13(100)$ \\
\hline Total & 29 & $2(7)$ & $1(3)$ & $26(90)$ \\
\hline \multicolumn{5}{|l|}{ Other newborn care ${ }^{d}$} \\
\hline Mothers & 138 & $2(2)$ & $10(7)$ & $126(91)$ \\
\hline Fathers & 7 & $0(0)$ & $0(0)$ & $7(100)$ \\
\hline Non-parental caregivers & 285 & $1(0.5)$ & $1(0.5)$ & $283(99)$ \\
\hline Total & 430 & $3(1)$ & $11(3)$ & $416(96)$ \\
\hline
\end{tabular}


towel before they carry my baby - Mother, primary facility

He is already dressed and covered with a towel so their hand will not touch the baby's skin - Mother, primary facility

\section{Discussion}

Our mixed methods exploratory study describes hand hygiene practices in the post-natal care ward, facility discharge and the home environment across six healthcare facilities in Nigeria. Our findings show a low prevalence of hand hygiene practice during post-natal care and in the home environment in the immediate post-birth period. Our study also provides data on the wide range of individuals who are involved in both maternal and newborn care along this continuum, including healthcare workers, cleaners, visitors, fathers, mothers, and non-parental caregivers. Not only were hand hygiene actions rare during our observation period, similarly to other studies, hand hygiene actions were largely inadequate; for example, $\mathrm{HCW}$ using gloves without having washed their hands with soap before [28, 32] and mothers and other caregivers rinsing hands with water only [21, 22, 33, 34]. Visitors in the health facility and non-maternal caregivers at the home accounted for the majority of observed hand hygiene opportunities, particularly around newborn care, but no hand hygiene actions were observed by these groups.

Handwashing with soap promotion will fail if inadequate infrastructure is in place. Unlike the labour and delivery rooms for facilities included in this study [29] the vast majority of post-natal care wards lacked adequate hand hygiene infrastructure and/or supplies. The lack of functioning hygiene infrastructure and supplies is commonly reported as a major barrier in both HCFs and at home to practicing hygienic behaviours [35-37]. The provision of handwashing facilities with soap at all points of care are the basic requirements for HCFs according to global monitoring strategies [38]. Point of care can be recognised as the place where the patient, the HCW, and the provision of care or treatment come together [12]. Our study shows that in the context of newborn care in the HCF, the 'point of care' should expand beyond delivery ward and include post-natal care areas. In the absence of hygiene infrastructure, alcoholbased hand rubs have been shown to improve hand hygiene practices and may be an effective low cost intervention for consideration [39-43].

The prevalence of appropriate hand hygiene by $\mathrm{HCW}$ during labour and delivery has been found to be generally low $[17,28,32,44]$. This study finds that $\mathrm{HCW}$ maintain inadequate hand hygiene practices into the post-natal care period. Increased emphasis on $\mathrm{HCW}$ washing hands with soap and appropriate glove use in post-natal care is needed and should be incorporated into standard quality of care and IPC improvement programs. Previous data from participating facilities shows that current models of step-down training on hand hygiene and IPC are inadequate, didactic, irregularly given and accompanied by little to no oversight [29]. In addition to general improvements to the overall infection control and hand hygiene training [41, 45-47], our data suggest that adherence to hand hygiene protocols specific to the post-natal care areas should be emphasised and integrated into multi-modal infection control strategies [41, 47].

The discharge process presents a valuable but underutilised opportunity to promote hand hygiene among all caregivers along the care continuum from facility to the home. Another study in Edo state, Nigeria found that mothers who practiced hygienic cord care reported that nurses had a stronger influence on mothers' behaviours compared to other caregivers [48]. Together with standardised discharge protocols and checklists [49], additional moments in the post-natal ward need to be identified to enable HCWs to provide and reinforce accurate, standardised, and simplified information in a way that it can be remembered and practiced by all caregivers while in the post-natal ward and at home.

Our observational study demonstrates the important role that non-maternal caregivers play during care both in the post-natal care ward and in the home environment. Other facility based studies in LMIC have documented the integral role of family members in patient management, their accompanying hand hygiene practices and the potential exposure risk they carry. For example, studies in Bangladesh reported that compliance of family members providing inpatient care ranged between $0 \%$ [50] and 3\% [34]. Studies on hygiene during neonatal care in the home environment focus primarily on the new mothers or birth attendants [18, 23, 24, 51]. Non-maternal caregivers are not only actively engaged in newborn care in these settings, but they are also important drivers of the mothers' handwashing behaviours $[21,22]$. Interventions may potentially overlook the critical role and engagement of fathers and extended family members in newborn contact [52]. In a tertiary hospital in India, Biswal et al. [53] reported a 13\% improvement of family member compliance following the implementation of a hand hygiene improvement strategy that included a caregiver-specific training component. Understanding the drivers of behaviours of the wider context within which the mother exists and how these behaviours are informed and modified by both the physical and social environment can help in the development of new interventions that target wider audiences in both the home and the healthcare [50, 52, 54]. 
The small number of facilities for this observational study limit the generalisability of our findings to beyond these study sites. Our study had a participant dropout rate of $26 \%$ prior to the home observations, which may have introduced bias into our study if the participants who dropped out systematically differed from those who remained or were later recruited into the study. However, data suggests that drop-outs and new enrolments were similar in age, previous births, and time spent travelling to clinic. Reactivity by participants to the presence of an observer may have led some actors to increase hand hygiene compliance [55]. However, this reactivity was minimised by avoiding any explicit mention of handwashing behaviour being the aim of the study and carrying out the observations before the household interviews and overall low levels of hand hygiene compliance observed in this study suggest that the impact of reactivity on handwashing behaviours was likely minimal.

\section{Conclusion}

Our study shows that hand hygiene along the entire continuum of maternal and newborn care is inadequate. In addition to the delivery room, future behaviour change interventions need to address hand hygiene practices within the post-natal care ward and early days at home and target a wider range of caregivers than mothers and healthcare workers. More in-depth research is required to understand the drivers of hand hygiene practices for all actors involved in maternal and newborn care in the immediate post-birth period and targeted interventions needed to improve hand hygiene practices developed. However, the basic provision of appropriate hygiene infrastructure in post-natal care wards is an urgent action that should prioritized as part of global efforts to expand water, sanitation, and hygiene coverage in healthcare facilities.

\section{Supplementary information}

Supplementary information accompanies this paper at https://doi.org/10. 1186/s12884-020-03282-3.

Additional file 1. Activities performed by non-maternal caregivers

\section{Abbreviations}

HCAl: Healthcare associated infections; HCF: Healthcare facilities; HCW: Healthcare workers; IPC: Infection prevention and control; IV: Intravenous; LMIC: LoW- and middle-income countries; WHO: World Health Organisation

\section{Acknowledgements}

We would like to give a very special thanks to the staff and management of participating HCFs for generously providing request information and the women who agreed to participate in this study without benefit to themselves. Special thanks go to the following institutions and individuals for their participation and support: Nigeria Federal Ministry of Health, Nigeria Federal of Water Resources, Kogi and Ebonyi State Ministries of Health, the Paediatric Association of Nigeria, the Nigerian Society of Neonatal Medicine, as well as other development partners. A very special thanks to the MCSP
Nigeria team for coordinating the study, and the special contributions of Abimbola Williams, Israel Ayegbusi and Patrick Ezeani and the Maternal and Child Survival Program (MCSP) teams in Nigeria and the USA for their technical contributions and support to the overall planning specifically, Adetiloye Oniyire, Chibugo Okoli, Emmanuel Adung, Emmanuel Ugwa, Gabriel Alobo, Gladys Olisaekee, Alyssa Om'Iniabohs, Ayne Worku, lan Moise, Joseph de Graft-Johnson, Obianuju Igbokwe, Olayinka Umar-Farouk, Soyannwo Tolulope and Vivian Obiama. All views expressed in this manuscript are those of the authors and do not necessarily reflect those of the funding organizations or affiliated institutions.

\section{Authors' contributions}

Conceptualization, EF, OC, OO and RD; methodology, HB, EF, OC and RD; validation, $\mathrm{HB}, \mathrm{OO}$, SS, and $\mathrm{RD}$; formal analysis, $\mathrm{YN}, \mathrm{HB}$ and $\mathrm{RD}$; investigation, $\mathrm{HB}$ and $\mathrm{OO}$; resources, $\mathrm{OO}$ and $\mathrm{SS}$; data curation, $\mathrm{HB}$; writing - original draft preparation, $\mathrm{YN}$; writing - review and editing, $\mathrm{YN}, \mathrm{HB}, \mathrm{EF}, \mathrm{OO}, \mathrm{OC}$, SS and RD. All authors have read and approved the final version of the manuscript.

\section{Funding}

This study was made possible by the generous support of the American people through the United States Agency for International Development (USAID) under the terms of the Cooperative Agreement AID-OAA-A-1400028. The contents are the responsibility of the Maternal and Child Survival Program and do not necessarily reflect the views of USAID or the United States Government.

\section{Availability of data and materials}

The datasets used and/or analysed during this study are available from the corresponding author on reasonable request.

\section{Ethics approval and consent to participate}

Ethics approvals were granted by the Institutional Review Board at London School of Hygiene and Tropical Medicine (Ref: 13643), and the ethical review boards of Kogi state (Ref: MOH/KGS/1376/1/84) and Ebonyi state (Ref: SMOH/ ERC/33/017). All participants provided written informed consent prior to participation. Mothers were only approached and enrolled prior to entering the second stage of labour. Women below the age of 18 years and/or with pre-existing conditions associated with a high-risk pregnancy and/or birth complications were excluded from the study.

\section{Consent for publication}

Not applicable.

\section{Competing interests}

The authors declare that they have no competing interests.

\section{Author details}

'Disease Control Department, London School of Hygiene and Tropical Medicine, London WC1E 7HT, UK. ${ }^{2}$ Infection and Immunity, South Australian Health and Medical Research Institute, Adelaide 5000, Australia. ${ }^{3}$ Maternal and Child Survival Program (MCSP)/Save the Children Nigeria, Abuja, Nigeria. ${ }^{4}$ Maternal and Child Survival Program (MCSP)/Save the Children US, Washington, DC 20036, USA.

Received: 8 June 2020 Accepted: 24 September 2020

Published online: 06 October 2020

References

1. Ganatra HA, Stoll BJ, Zaidi AK. International perspective on early-onset neonatal sepsis. Clin Perinatol. 2010;37(2):501-23.

2. Leach A, McArdle TF, Banya WA, Krubally O, Greenwood AM, Rands C, et al. Neonatal mortality in a rural area of the Gambia. Ann Trop Paediatr. 1999; 19(1):33-43.

3. Zaidi AK, Huskins WC, Thaver D, Bhutta ZA, Abbas Z, Goldmann DA Hospital-acquired neonatal infections in developing countries. Lancet. 2005; 365(9465):1175-88.

4. Zaidi AKM, Thaver D, Ali SA, Khan TA. Pathogens associated with sepsis in newborns and young infants in developing countries. Pediatr Infect Dis J. 2009;28(1):S10-S8 
5. Campbell OM, Calvert C, Testa A, Strehlow M, Benova L, Keyes E, et al. The scale, scope, coverage, and capability of childbirth care. Lancet. 2016; 388(10056):2193-208.

6. Hussein J, Mavalankar DV, Sharma S, D'Ambruoso L. A review of health system infection control measures in developing countries: what can be learned to reduce maternal mortality. Glob Health. 2011;7:14.

7. Montagu D, Sudhinaraset M, Diamond-Smith N, Campbell O, Gabrysch S, Freedman $L$, et al. Where women go to deliver: understanding the changing landscape of childbirth in Africa and Asia. Health Policy Plan. 2017;32(8):1146-52.

8. WHO. Essential newborn care: report of a technical working group, Trieste, 25-29 April 1994. Geneva: Maternal and Newborn Health, Safe Motherhood, World Health Organization, Family and Reproductive Health; 1996.

9. Blencowe H, Cousens S, Mullany LC, Lee AC, Kerber K, Wall S, et al. Clean birth and postnatal care practices to reduce neonatal deaths from sepsis and tetanus: a systematic review and Delphi estimation of mortality effect. BMC Public Health. 2011;11(Suppl 3):S11.

10. Luangasanatip $N$, Hongsuwan M, Limmathurotsakul D, Lubell $Y$, Lee AS, Harbarth S, et al. Comparative efficacy of interventions to promote hand hygiene in hospital: systematic review and network meta-analysis. BMJ. 2015:351:h3728.

11. Storr J, Twyman A, Zingg W, Damani N, Kilpatrick C, Reilly J, et al. Core components for effective infection prevention and control programmes: new WHO evidence-based recommendations. Antimicrob Resist Infect Control. 2017;6(1):6.

12. $\mathrm{WHO}$. A guide to the implementation of the $\mathrm{WHO}$ multimodal hand hygiene improvement strategy. 2009. Available at: http://whqlibdoc.who.int/ hq/2009/WHO_IER_PSP_2009 02_eng.pdf. 2011.

13. WHO. Guidelines on core components of infection prevention and control programmes at the national and acute health care facility level. Geneva: World Health Organization; 2016.

14. $\mathrm{WHO}, \mathrm{WHO}$ Patient Safety. WHO guidelines on hand hygiene in health care Geneva: World Health Organization; 2009. [updated 2009. Available from: https://apps.who.int/iris/handle/10665/44102.

15. WHO, UNICEF, UNFPA. Pregnancy, childbirth, postpartum, and newborn care: a guide for essential practice. Geneva: World Health Organization; 2003

16. Cohen B, Saiman L, Cimiotti J, Larson E. Factors associated with hand hygiene practices in two neonatal intensive care units. Pediatr Infect Dis J. 2003;22(6):494-9.

17. Danda G, Dube K, Dube P, Mudokwenyu-Rawdon C, Bedwell C. An observational study of midwives' practices to prevent peripartum sepsis in Zimbabwe. Afr J Midwifery Womens Health. 2015;9(1):17-21.

18. Darmstadt GL, Hussein MH, Winch PJ, Haws RA, Gipson R, Santosham M. Practices of rural Egyptian birth attendants during the antenatal, intrapartum and early neonatal periods. J Health Popul Nutr. 2008;26(1):36-45.

19. Lam BC, Lee J, Lau YL. Hand hygiene practices in a neonatal intensive care unit: a multimodal intervention and impact on nosocomial infection. Pediatrics. 2004;114(5):e565-71.

20. Picheansathian W, Pearson A, Suchaxaya P. The effectiveness of a promotion programme on hand hygiene compliance and nosocomial infections in a neonatal intensive care unit. Int J Nurs Pract. 2008;14(4): 315-21.

21. Greenland K, Iradati E, Ati A, Maskoen YY, Aunger R. The context and practice of handwashing among new mothers in Serang, Indonesia: a formative research study. BMC Public Health. 2013;13(1):830.

22. Parveen S, Nasreen S, Allen JV, Kamm KB, Khan S, Akter S, et al. Barriers to and motivators of handwashing behavior among mothers of neonates in rural Bangladesh. BMC Public Health. 2018;18(1):483.

23. Ram PK, Nasreen S, Kamm K, Allen J, Kumar S, Rahman MA, et al. Impact of an intensive perinatal handwashing promotion intervention on maternal handwashing behavior in the neonatal period: findings from a randomized controlled trial in rural Bangladesh. Biomed Res Int. 2017;2017:6081470.

24. Darmstadt GL, Syed U, Patel Z, Kabir N. Review of domiciliary newborn-care practices in Bangladesh. J Health Popul Nutr. 2006;24(4):380-93.

25. Rhee V, Mullany LC, Khatry SK, Katz J, LeClerq SC, Darmstadt GL, et al. Maternal and birth attendant hand washing and neonatal mortality in southern Nepal. Arch Pediatr Adolesc Med. 2008;162(7):603-8.

26. WHO. WHO technical consultation on postpartum and postnatal care Geneva: World Health Organization; 2010. [updated 2010. Available from: https://apps.who.int/iris/handle/10665/70432.
27. WHO. WHO recommendations on postnatal care of the mother and newborn. Geneva: World Health Organization; 2014. [updated 2014 Available from: https://apps.who.int/iris/handle/10665/97603.

28. Buxton H, Flynn E, Oluyinka O, Cumming O, Esteves Mills J, Shiras T, et al. Hygiene during childbirth: an observational study to understand infection risk in healthcare facilities in Kogi and Ebonyi States, Nigeria. Int J Environ Res Public Health. 2019;16(7)

29. Buxton H, Flynn E, Oluyinka O, Cumming O, Esteves Mills J, Shiras T, et al. Barriers and opportunities experienced by staff when implementing infection prevention and control guidelines during labour and delivery in healthcare facilities in Nigeria. J Hosp Infect. 2019;103(4):428-34.

30. Sax H, Allegranzi B, Uçkay I, Larson E, Boyce J, Pittet D. 'My five moments for hand hygiene': a user-centred design approach to understand, train, monitor and report hand hygiene. J Hosp Infect. 2007;67(1):9-21.

31. Ditai J, Abeso J, Odeke NM, Mobbs N, Dusabe-Richards J, Mudoola M, et al. BabyGel pilot: a pilot cluster randomised trial of the provision of alcohol handgel to postpartum mothers to prevent neonatal and young infant infection-related morbidity in the community. Pilot Feasibility Stud. 2019:5:49.

32. Gon G, de Bruin M, de Barra M, Ali SM, Campbell OM, Graham WJ, et al. Hands washing, glove use, and avoiding recontamination before aseptic procedures at birth: a multicenter time-and-motion study conducted in Zanzibar. Am J Infect Control. 2019;47(2):149-56.

33. Curtis VA, Danquah LO, Aunger RV. Planned, motivated and habitual hygiene behaviour: an eleven country review. Health Educ Res. 2009;24(4): 655-73.

34. Horng LM, Unicomb L, Alam MU, Halder AK, Shoab AK, Ghosh PK, et al. Healthcare worker and family caregiver hand hygiene in Bangladeshi healthcare facilities: results from the Bangladesh National Hygiene Baseline Survey. J Hosp Infect. 2016;94(3):286-94.

35. Bazzano AN, Oberhelman RA, Potts KS, Gordon A, Var C. Environmental factors and WASH practices in the perinatal period in Cambodia: implications for newborn health. Int J Environ Res Public Health. 2015;12(3): 2392-410.

36. Yawson $A E$, Hesse AA. Hand hygiene practices and resources in a teaching hospital in Ghana. J Infect Dev Ctries. 2013;7(4):338-47.

37. Devine J. Beyond tippy-taps: the role of enabling products in scaling up and sustaining handwashing. Waterlines. 2010:304-14.

38. WHO. WASH in health care facilities: global baseline report 2019. 2019.

39. Erasmus V, Daha TJ, Brug H, Richardus JH, Behrendt MD, Vos MC, et al. Systematic review of studies on compliance with hand hygiene guidelines in hospital care. Infect Control Hosp Epidemiol. 2010;31(3):283-94.

40. Nguyen KV, Nguyen PT, Jones SL. Effectiveness of an alcohol-based hand hygiene programme in reducing nosocomial infections in the urology Ward of Binh Dan Hospital, Vietnam. Tropical Med Int Health. 2008; 13(10):1297-302.

41. Ofek Shlomai N, Rao S, Patole S. Efficacy of interventions to improve hand hygiene compliance in neonatal units: a systematic review and metaanalysis. Eur J Clin Microbiol Infect Dis. 2015;34(5):887-97.

42. Ramasethu J. Prevention and treatment of neonatal nosocomial infections. Matern Health Neonatol Perinatol. 2017;3:5.

43. Uneke CJ, Ndukwe CD, Oyibo PG, Nwakpu KO, Nnabu RC, Prasopa-Plaizier N. Promotion of hand hygiene strengthening initiative in a Nigerian teaching hospital: implication for improved patient safety in low-income health facilities. Braz J Infect Dis. 2014;18(1):21-7.

44. Simbar M, Ghafari F, Zahrani ST, Majd HA. Assessment of quality of midwifery care in labour and delivery wards of selected Kordestan Medical Science University hospitals. Int J Health Care Qual Assur. 2009;22(3):266-77.

45. Charrier L, Allochis MC, Cavallo MR, Gregori D, Cavallo F, Zotti CM. Integrated audit as a means to implement unit protocols: a randomized and controlled study. J Eval Clin Pract. 2008;14(5):847-53.

46. Zingg W, Holmes A, Dettenkofer M, Goetting T, Secci F, Clack L, et al. Hospital organisation, management, and structure for prevention of healthcare-associated infection: a systematic review and expert consensus. Lancet Infect Dis. 2015;15(2):212-24.

47. Cherry MG, Brown JM, Bethell GS, Neal T, Shaw NJ. Features of educational interventions that lead to compliance with hand hygiene in healthcare professionals within a hospital care setting. A BEME systematic review: BEME guide no. 22. Med Teach. 2012;34(6):e406-20.

48. Abhulimhen-lyoha BI, Ibadin MO. Determinants of cord care practices among mothers in Benin City, Edo State, Nigeria. Niger J Clin Pract. 2012; 15(2):210-3 
49. Spector JM, Agrawal P, Kodkany B, Lipsitz S, Lashoher A, Dziekan G, et al. Improving quality of care for maternal and newborn health: prospective pilot study of the WHO safe childbirth checklist program. PLoS One. 2012; 7(5):e35151.

50. Islam MS, Luby SP, Sultana R, Rimi NA, Zaman RU, Uddin M, et al. Family caregivers in public tertiary care hospitals in Bangladesh: risks and opportunities for infection control. Am J Infect Control. 2014;42(3):305-10.

51. Bazzano AN, Taub L, Oberhelman RA, Var C. Newborn care in the home and health facility: formative findings for intervention research in Cambodia. Healthcare. 2016;4(4):94.

52. Comrie-Thomson L, Tokhi M, Ampt F, Portela A, Chersich M, Khanna R, et al. Challenging gender inequity through male involvement in maternal and newborn health: critical assessment of an emerging evidence base. Cult Health Sex. 2015;17(Suppl 2):S177-S89.

53. Biswal M, Angrup A, Rajpoot S, Kaur R, Kaur K, Kaur H, et al. Hand hygiene compliance of patients' family members in India: importance of educating the unofficial 'fourth category' of healthcare personnel. J Hosp Infect. 2020; 104(4):425-9.

54. Barker G, Ricardo C, Nascimento M. Engaging men and boys in changing gender-based inequity in health: evidence from programme interventions. 2007. Geneva: World Health Organization; 2009.

55. Ram PK, Halder AK, Granger SP, Jones T, Hall P, Hitchcock D, et al. Is structured observation a valid technique to measure handwashing behavior? Use of acceleration sensors embedded in soap to assess reactivity to structured observation. Am J Trop Med Hyg. 2010;83(5):1070-6.

\section{Publisher's Note}

Springer Nature remains neutral with regard to jurisdictional claims in published maps and institutional affiliations.

Ready to submit your research? Choose BMC and benefit from:

- fast, convenient online submission

- thorough peer review by experienced researchers in your field

- rapid publication on acceptance

- support for research data, including large and complex data types

- gold Open Access which fosters wider collaboration and increased citations

- maximum visibility for your research: over $100 \mathrm{M}$ website views per year

At BMC, research is always in progress.

Learn more biomedcentral.com/submissions 